\title{
Stendhal picaresque? Tours et détours d'écriture
}

\section{Rita Zaffarami Berlenghini}

\section{(2) OpenEdition}

\section{Journals}

Édition électronique

URL : http://journals.openedition.org/studifrancesi/27031

DOI : 10.4000/studifrancesi.27031

ISSN : 2427-5856

Éditeur

Rosenberg \& Sellier

Édition imprimée

Date de publication : 31 décembre 2006

Pagination : 478-495

ISSN : 0039-2944

\section{Référence électronique}

Rita Zaffarami Berlenghini, «Stendhal picaresque? Tours et détours d'écriture », Studi Francesi [En ligne], 150 (L | III) | 2006, mis en ligne le 30 novembre 2015, consulté le 08 novembre 2020. URL : http://journals.openedition.org/studifrancesi/27031; DOI : https://doi.org/10.4000/studifrancesi. 27031

\section{(c) $(1)$}

Studi Francesi è distribuita con Licenza Creative Commons Attribuzione - Non commerciale - Non opere derivate 4.0 Internazionale. 


\section{Stendhal picaresque? Tours et détours d'écriture}

Ce point d'interrogation ajouté au titre ne révèle que quelques hésitations à propos de la solidité d'une thèse concernant une véritable ascendance picaro-espagnole des récits stendhaliens presque tous inachevés - tels que Le Philtre, A-Imagination, Le Juif, Don Pardo, Le Coffre et le revenant, Le Chevalier de Saint Ismier et Ménuel, court récit, celui-ci, qui est inséré dans Lucien Leuwen.

Faute de preuves, de preuves éclatantes, on espère trouver des résultats dans les différentes tonalités de ce point d'interrogation auquel on confie les nuances de la pensée. À cela on peut ajouter des «retentissements» évocatoires lesquels, en étayant cette idée de la présence de l'Espagne picaresque, vont se joindre à quelques données objectives. Tout cela devrait témoigner d'une orientation littéraire que Stendhal semble révéler surtout dans les années trente.

Voyons d'abord ses lectures: dans sa bibliothèque de Civitavecchia il possédait La Vie de Guzman d'Alfarache de Mateo Alemán; il avait lu Le Toledan; Don Quichotte avait été son livre de chevet dès la découverte qu'il en avait fait dans la bibliothèque de Claix (et dont le thème du voyage, thème typiquement picaresque, l'avait profondément frappé); Gil Blas aussi avait été une autre lecture importante. Enfin, il aimait beaucoup le théâtre de Lope de Vega ${ }^{1}$.

Dans la dernière décennie de sa vie Stendhal semble se pencher vers cette Espagne du Mal, une Espagne littéraire où les actions des protagonistes des romans ne peuvent avoir qu'un seul style, celui de la rapidité. Stendhal se laisse entraîner! La rapidité implique la phrase hachée et nerveuse, apte à la description d'un vol ou d'un meurtre. La phrase rapide, sèche, répond à la rapidité de l'action: le crime et le vol sont toujours perpétrés rapidement, les gestes consistent en démarrages déclanchant la fuite après le vol. La fuite vers quoi? Vers d'autres vols... peut-être! Il est vrai que la phrase sèche est typiquement stendhalienne (les dernières pages du Rouge en sont un exemple évident), mais ici, dans ces récits, ce genre de phrase semble jouer un rôle capital. Aucune concession donc à la musique du cœur. Autant qu'il est permis de juger et d'interpréter, on voudrait souligner ceci: les romans picaresques espagnols pourraient avoir eu leur importance en tant que modèles permettant à l'écrivain de s'évertuer à rejoindre une littérarité où fond et forme strictement liés, profondément unis, créent un syncrétisme de valeurs. Mais il y a plus à vrai dire: Stendhal semble se consacrer à une élaboration d'écriture inexpérimentée jusque-là, car c'est une forme revêtant des faits et non des idées. Un style qui oblige à raconter des faits, et qui ne permet pas à l'écrivain de se complaire dans les analyses psychologiques. Les affres du style avaient souvent gêné Stendhal, mais de façon puérile et bizarre, une étrange «maladie» qu'il «guérissait» par ses mélanges de langues différentes, sorte d'idiolecte qu'il pratiquait, peut-être, au cours de ses monologues intérieurs. Autrefois c'était le dessin! Une forme d'expression très éloquente où la mimique, à peine brossée, et les jeux de physionomie offraient un langage génial, parfois abstrus dans son iconicité (v. l'autoportrait, brossé par Stendhal, au cours d'un voyage dans le Midi avec George Sand et Alfred de Musset). Il est évident que Stendhal ici se révèle ambigu et aléatoire: «Je ne sais où je vais », écrit-il, presque en exergue, dans le plan de $A$-Imagination, après la date du 2 août 1838. L'ambiguïté et le hasard: est-ce que Stendhal va

(1) V. Del Litto, La Vie intellectuelle de Stendhal, Paris, P.U.F. 1959 , p. 132. Stendhal arrivera à préférer Les Castelvins et les Montezes de Lope de Vega à Romeo et Juliette de Shakespeare. 
se lancer dans l'incertitude, dans l'inconnu? On ne peut pas dire! Ce qui est certain c'est qu' il cherche ... il cherche avidement mais peut-être un peu aveuglement. Malgré cela il avait écrit - et il a continué à écrire - des pages mémorables où le lyrisme du bonheur est la sève de son art. Mais dans ces récits des années trente, rien de rien, le lyrisme est banni... Stendhal avait toujours prôné la sécheresse du Code Civil, le tour froid et le ton positif, il avait soutenu qu'il fallait marcher droit au but (v. La Vie de Henry Brulard), eh bien! Dans ces récits il donne la preuve la plus tangible de la réalisation de ces sentences dogmatiques.

La tendance à créer les dénouements rapides dans le développement du récit - ce qui est totalement en accord avec la phrase sèche et nerveuse - était déjà présente dans les romans. Mais ici Stendhal imagine et crée le choc, clôt le récit de façon abrupte, l'allure subit une interruption, les dénouements sont très nombreux, beaucoup plus que jadis. Il circonscrit l'action à la situation du moment, par conséquent «l'iconicité», c'est-à-dire le langage le plus touchant, produit ses effets: on lit et on voit. Ces récits présentent plusieurs irregularités où prolifèrent notes et fragments en un tout inorganique... mais qui semble attendre son achèvement. Un ton d'allegresse émerge malgré tout de ces pages, c'est la «felicità dello scrivere» dont parlait souvent Leonardo Sciascia à propos de l'écriture chez Stendhal.

Malgré la sécheresse, toujours prônée, l'écrivain révèle ici une intensité protéiforme des signifiés. Ce «dire» permet de «voir» et éveille des sensations, parfois il semble évoquer même des motifs rythmiques.

«Robert à quatorze ans est un petit coquin complet quant au cœur [...]

Robert serait serviteur de M. l'abbé Richet, jeune prêtre de vingt-huit ans qui cherchait à se faire un nom par l'éloquence de la chaire. Il est vrai que Robert ne savait pas un mot d'ortographe; mais il était audacieux, grand, bien découpé, et ces fonctions de secrétaire consisteraient à cirer les souliers de l'abbé et à l'accompagner le soir»².

Autrefois, dans les notes marginales de Don Pardo on lit:

«Le capucin veut dépayser cette petite peste et l'emmène au couvent d'Orvieto. Pardo sent que le capucin est son ennemi, il veut se faire un protecteur.

Un capucin ennemi de l'autre et qui a essayé de plaire à la fermière, le fait jaser...lui enseigne d'aller à la grange où sur le foin la fermière donne des rendez-vous muets (entourée qu'elle est des sept ou huit garçons de la ferme) au père Egregio, premier capucin. Francesco, le second capucin, a ainsi la fermière» ${ }^{3}$.

Indiscrétions savoureuses de celui qui raconte! Tableau captivant pour le lecteur qui «regarde»! L'image, issue de la parole, impressionne le lecteur. La forme «visuelle» évoque et donne naissance à des créations en leur prêtant existence et continuité.

Le renouvellement de la forme joue son rôle capital, même si cela ne fait que fortifier des tendances déjà existantes. Il faut ajouter que Stendhal n'a jamais respecté cette fusion - contenu forme - aussi rigidement. Maintenant cela semble devenir son idée fixe. Il ne cesse pas d'insister sur son projet; il aime ces thèmes, il ne voudrait pas les abandonner, il corrige, il y revient, il se sermonne. C'est à cette fusion - et à sa ténacité d'écrivain - qu'on doit la visualisation des scènes. La puissance visuelle est le produit de cette fusion: visualiser c'est peindre, l'écrivain brosse une société de fripons, rusés ou idiots, en même temps victimes et bourreaux.

Des personnages ratés? Oui et non! Il faut admettre qu'ils sont inoubliables. Pourquoi? Parce qu'ils sont éloquents. Leurs histoires demeurent intactes, malgré les interruptions et les remaniements. On ne les oublie pas, elles subsistent ..... de là leur

(2) STEndHAL, A-Imagination in Le Rose et le Vert, Mina de Vanghel et autres nouvelles, Paris,
Gallimard, 1982, p. 332.

(3) Op. cit., p. 538 
validité. À cela il faut ajouter l'importance des détails: ces récits sont circonstanciés car, ici, tels que des pilotis, les détails ne font qu'étayer le réalisme de la description. Ce qui est descriptible offre un «rendement» précieux au profit de l'efficience du texte. Est-ce l'ivresse de l'écriture? Une écriture concise, segmentée et qui séduit l'écrivain, mais contre laquelle il bute. Le sujet aussi le séduit. Mais, lequel des deux, objectera-t-on, est-il le plus envoûtant? Séparés ni l'un ni l'autre, naturellement! C'est la fusion stricte des deux éléments qu'il veut rejoindre! Cela est sûr... mais c'est ici le problème... il tâtonne, il refait, il s'égare... «je ne sais où je vais»... il reprend le thème... et puis souvent c'est le néant de l'inachevé.

Après une comparaison entre quelques pages de Guzman d'Alfarache et d'autres de A-Imagination (ou de Don Pardo, ou Le Philtre ou Le Juif) y compris les plans, il faut dire que si le style de l'auteur espagnol possède une allure svelte, et si la phrase est d'une agilité incomparable, elle n'est pas sèche, elle est colorée, riche en valeurs adjectivales. Les actions, tout en s'échelonnant rapidement, sont détaillées, par conséquent leur intensité sémantique en est enrichie. On n'a pas, ici, un style dépouillé, on y trouve la rapidité unie à une expressivité surprenante. La tonalité des adjectifs de qualité est éloquente. L'usage des proverbes, usage assez fréquent - ainsi que celui des allusions ou des sous-entendus - donne de la vigueur à l'axe syntagmatique. La phrase est nerveuse, mais non dépouillée.

Stendhal, au contraire, écrit des pages beaucoup moins «succulentes», ses récits sont beaucoup moins riches en couleurs... mais à l'agilité il unit la simplicité de ce qui est spontané. Les bizarreries d'un contenu, inusuel chez lui, fusionnent magnifiquement en une forme simple, spontanée, apte à la situation. Les phrases rapides et les ellipses - l'a-t-on déjà remarqué - sont beaucoup plus nombreuses que jadis. Sont elles des exercices de style? En s'inspirant des exploits dérivant, plus ou moins, des contenus picaresques, Stendhal n'arrive-t-il pas à créer plus efficacement, le style nerveux, haché où le jargon et le parler argotique lui offrent leur utile support?

Le style de Mateo Alemán appartient aux gueux et, curieusement, aux clercs aussi. Il est agile et rapide, riche et coloré, il fait songer à la langue des Gitans. Parfois c'est le parler régional de toute une pègre loqueteuse qui arrive à s'estropier pour recevoir l'aumône, ou bien c'est l'histoire des passe-volants, faux soldats qui essaient d'obtenir la solde ${ }^{4}$. Ces thèmes et cette langue ne pouvaient que fasciner Stendhal. Toutefois on ne veut pas établir ici une contiguïté stricte entre le picarisme et le dernier Stendhal (il existe, paraît-il, une lignée picaresque, plus ou moins acceptée comme telle, qui va de Simplicissimus à Gil Blas, de Moll Flanders à Jacques le Fataliste; peut être pourrait-on insérer aussi les récits stendhaliens dont on est en train de parler, et même Lamiel à cause de l'allure du roman et de certains traits caractérisant cette «fille du diable»).

En relisant ces récits on n'y trouve pas, certes, une contiguïté exacte, mais des échos de la picaresque, des échos importants qui font réfléchir, indéniablement captivants et dont la suggestion provient d'un monde quasi exotique qui devait attirer l'écrivain français, d'autant plus que son penchant pour les sujets horrifiques n'est pas à négliger, ainsi que sa tendance à conférer à certains de ses héros cet air de vagabonds et ce ton d'interminables errances qui les caractérisent; les vagabondages vont de pair avec l'allure de certains romans où les déplacements créent une atmosphère qui est propre aux romans picaresques. Le langage est adéquat.

Les années trente: Stendhal est à son apogée et puisque... inquietum est cor nostrum... il cherche encore d'autres contenus et une forme presque inédite, dont l'ori- 
gine, probablement, est à découvrir dans le passé (le Stendhal du style de Code Civil qu'il prônait, qu'il désirait pratiquer... mais qu'il n'a jamais totalement réalisé) et dont l'essor est dans ces récits. C'est un essor écrasé brutalement et fatalement par le néant! Toutefois la mort, qui tout retranche, ne peut pas tout anéantir car ces ébauches (bien que telles) rendent hommages au talent de l' écrivain. Il aimait ces sujets, cela est sûr. Jean Prévost a écrit: «Stendhal ne décrit bien que ce qu'il préfère!». Pardo ou le Robert de A-Imagination devaient lui plaire beaucoup. Encore une fois c'est une écriture iconique, formellement déficitaire, mais sans faiblesse dans son ossature. C'est une écriture sèche et forte. Ces récits sont des ébauches remarquables dans leur genre.

$$
* * *
$$

Dans Guzman d'Alfarache il y a ce jeu de miroirs qui exprime la duplicité de l'âme humaine dans ses différentes nuances! Mateo Alemán présente deux Guzman «qui se donnent la réplique [...] l'un est un pécheur, l'autre un théologien et un ascète [...] celui-ci naît des dépouilles de celui-là et se contemple tel qu'il fut [...] tout Guzman d'Alfarache est dans ce jeu de miroirs: roman dialectique où tout est contraste, opposition de plus à moins» ${ }^{5}$. Dans A-Imagination et dans Don Pardo on retrouve ce même jeu de miroirs: Robert (lequel aurait dû être le reflet de Robert Macaire) n'est que l'image inverse de l'abbé Richet. Lorsque ce «miroir» agit tout se défigure, le reflet déforme le «sujet». Celui-ci, ayant perdu son identité, vit virtuellement. C'est ici qu'on a le passage le plus important du récit, il mériterait une étude à part. Dans ce récit, en outre il y a un curieux pastiche entre métamorphose et métaphore, où les termes concrets contiennent l'abstraction dérivée de la transformation (v. plans). (Par exemple dans A-Imagination on lit ... Robert a une «physionomie d'apprenti perruquier ... L'air faux et presque menteur empêchait cette physionomie d'avoir l'air commun»).

Pas trop de sensations, sinon les plus élémentaires chez les personnages créés par Stendhal. Elles sont rares, chez les protagonistes des romans picaresques aussi tels que Guzman d'Alfarache (ou Lazare de Tormes, ou El Buscón de Quevedo). Ces derniers sont totalement conditionnés par leurs situations, on dirait qu'ils n'ont pas d'âme. Mais ici les réflexions de l'auteur ne manquent pas: il y en a, elles sont profondes bien que synthétiques. Ces malchanceux ont une mobilité innée (de là l'importance du thème du voyage qui est typiquement picaresque). Ils se déplacent souvent, ils n'ont pas toujours la possibilité de manger à leur faim. Le picaro est toujours affamé, il ne mange pas, il dévore, sa voracité fait partie de ses contractions musculaires. (Certains personnages stendhaliens, par exemple Don Pardo ou le Robert de A-Imagination, ont ces mêmes habitudes). Le picaro est insensible aux passions nobles! Il en est de même devant les humiliations où il ne révèle pas de réactions: pour lui, esquiver c'est réagir, l'escamotage est sa seule forme de réaction. Les allusions sont sa force, on trouve les mêmes allusions dans Guzman d'Alfarache et dans El Buscón de Quevedo. «Couronner de papier» c'est une allusion à la mitre de papier dont on coiffait les hérétiques pour le supplice. Mateo Alemán et Francisco de Quevedo appartiennent à la même culture, ils ont les mêmes formules de locution, car la pensée est souvent la même et l'allusion va de pair avec l'insinuation.

La vitalité du picaro est au service de sa propre survivance, le contraste entre la psychologie du personnage et l'exigence de survie, se neutralise puisque la psychologie disparait car elle est annulée par l'exigence même de survivre. Voilà pourquoi le 
picaro se débat tel qu'un pantin sans âme... Un pantin! Ce protagoniste est traité et exploité, par son auteur, en tant que personnage-type et non en tant que personnage doué de son identité et de son altérité. Cela ne fait que limiter le rôle de ces jeunes vagabonds sympatiques et malheureux. Ils sont trop collés à leurs clichés. Dommage! Les héros-protagonistes stendhaliens sont beaucoup moins stéréotypés, ou mieux ils ne le sont point: le Filippo de Le Juif ne ressemble pas à Pardo et tous les deux diffèrent énormément du Robert de $A$-Imagination etc. Ce sont les traits extérieurs qui les rapprochent, et c'est l'art de Stendhal qui les anime.

Le milieu picaresque est donc celui des escrocs, des meurt-de-faim, des banqueroutiers qui vivent et agissent à côté des marchands, magistrats, seigneurs et clercs protégeant, apparemment, les jeunes gens.

La Vie de Guzman d' Alfarache peut avoir frappé le Stendhal avide de connaissance et le lecteur gourmand. Dans ce roman on a affaire à des personnages dont les gestes et la poussée des instincts, semblent se concentrer pour créer tout un univers d'intrigues et de noirceur. Ce sont des personnages doubles, maîtres en l'art de feindre, dont les sourires narquois se confondent avec la sournoiserie la plus ingénieuse.

Il ne manque pas les monstres, par exemple celui de Ravenne qui était «...sans bras à la place desquels nature lui avait posé deux ailes de chauve-souris. Au milieu de la poitrine il portait l'y de Pythagore et une + bien dessinée entre l'estomac et le ventre. Il était hermaphrodite... $\gg^{6}$. C'est horripilant et en même temps très intéressant: on pourrait supposer une liaison entre ce monstre et un certain aspect du clergé corrompu ou bien avec la dépravation des «grosses légumes» de la société. Cela ne pouvait qu'attirer l'auteur de Les Cenci.

Un lien semble donc exister entre ce genre picaresque et les récits stendhaliens tels que Don Pardo, A-Imagination, Le Juif, Le Philtre. (Les autres récits ci-dessus cités et qui remontent toujours aux années trente, présentent des reflets un peu moins intéressants par rapport aux romans picaresques, mais ils ne sont pas à négliger car ce sont des reflets toujours à rapporter au milieu espagnol. On les analysera par la suite).

Le Philtre (1830), Le Juif (1831), A-Imagination (1838), Don Pardo (1840) dans ces petits chefs-d'œuvre (petits prodiges de l'intelligence, surtout A-Imagination et Don Pardo malgré leur état d'ébauche) on n'a que faim, horreur, manigances, métamorphoses et métaphores, vols et voleurs-volés et enfin ... judaïté et bâtardise (qu'on n'oublie pas ce Guzman faisant souvent allusion à son père bâtard, juif mal converti et sodomite). Dans A-Imagination on fait la connaissance d'un ami de Robert qui s'appelle Carière: en quelques lignes Stendhal brosse ce petit personnage, ce bâtard «honnête en grande partie par défaut d'esprit», comique, patibulaire, mais rusé autant qu'il suffit.

La judaïté et la bâtardise représentent la «condition humaine» la plus désolante: «l'homme est ce qu'il cache... un misérable petit tas de secrets», écrira beaucoup plus tard André Malraux. Tous ces personnages cachent leurs vies jusqu'au moment où ils ne naissent à une vie plus étendue, à la vie littéraire. Auparavant ils existaient dans la réalité historique ainsi que dans d'autres domaines littéraires. Mais, ici, lorsque l'écrivain les fait parler, ils se délivrent de leurs liens en révélant leur tas de secrets tout en se bornant aux allusions ou aux sous - entendus... et la misère est là, elle les attend. Le lecteur y déniche une vérité historique: ils sont obligés de tomber dans le mal. Ces êtres minables, profondément honnêtes, sont prêts à nuire, mais leurs actions délétères ne se heurtent pas à leur fond d'honnêteté. Pourquoi? La réponse est aux moralistes espagnols (ici il faut circonscrire notre réflexion à l'Espagne du 
$\mathrm{XVII}^{\mathrm{e}}$ siècle et non au Stendhal des récits courts vu que la tonalité de ses écrits est ici libre, enjouée, patibulaire et désinvolte en même temps, mais jamais moralisante). Les moralistes cléricaux espagnols donc: on a déjà laissé entendre l'imposante masse de travail auquel le clergé se soumettait pour instruire la jeunesse en faisant œuvre de moralisation. Il fallait, à tout prix, édifier les mœurs des gens pauvres. Les misérables, n'ayant d'autre choix que la débauche, devaient être considérés comme des victimes désignées dès leur naissance. Le clergé probablement réfléchissait avec sincérité sur les dévergondages de la jeunesse: les jeunes, pensait-on, étant condamnés à la débauche étaient justifiés, leur fond naturel d'honnêteté demeurait intact, mais inactif. À cela il faut ajouter l'anticléricalisme (auquel ne seraient pas tout à fait étrangers certains religieux radicalement attachés à la plus stricte authenticité de leurs croyances) qui couvait depuis l'humanisme propagé par les disciples d'Erasme et qu'on retrouve assez ostensiblement dans La vie de Lazare de Tormes.

Les Capucins et les vieux moines qui attirent le Pardo stendhalien, ce polisson de Civitavecchia, devenu entre temps lui aussi moine de Tiers Ordre, se consacrent aux pratiques d'une homosexualité et d'une pédérastie aberrantes. (v. plan) Ici Stendhal, lorsqu'il décrit ces scènes de sodomie triomphante, emploie un parfait italien bien seyant à la vulgarité. C'est du satanisme! Et les tartuffes sont toujours là. Aucune réflexion de la part de l'auteur. Le réalisme se confond avec l'irréalité des situations, la vision du réel en est presque déformée comme à travers une lentille grossissante. Stendhal n'envahit pas la sphère de l'abstrait, Stendhal n'est pas Balzac. Tout est circonscrit autour du réalisme de la situation, tout élément cérébral, hallucinatoire, tendant à l'abstraction y est exclu. Balzac, au contraire, brossant les différentes images de Vautrin ne peint pas un personnage cynique mais le cynisme, père Grandet n'est pas un avare, il est l'incarnation de l'avarice... et l'or, c'est la tyrannie de l'or rongeant l'homme.

Le mal que décrit Stendhal dans ces récits - à ce stade d'ébauche - n'a pas d'implications d'ordre moral ou moralisant, alors que dans les romans picaresques, on souligne remarquablement l'existence du mal: la méchanceté du picaro n'est pas à combattre mais à cultiver, c'est ainsi que l'on peut mieux exploiter la jeune victime! C'est le moraliste qui souligne le mal de façon brutale et effrayante! Il a aussi tendance à indiquer le Bien par l'entremise du Mal. Ce milieu picaresque est un univers mythique contenant le mythe du Mal, il s'agit d'une mythologie inverse par rapport aux images idéalisées des romans de chevalerie espagnols dont «l'humanité est portée à son plus haut degré de perfection»? Le Mal, quelle force d'attraction pour l'auteur de Les Cenci!

C'est une période où Stendhal lui-même avoue le tarissement de sa créativité (mais qu'on n'oublie pas qu'à l'époque il écrit: «n'ayant rien à lire j'écris», phrase celle-ci assez significative des tours et détours d'écriture qu'il va révéler et de la passion aussi qui l'envahit devant la page). En plus c'est la période où il écrit Le Rouge (1830), Les Souvenirs d'Egotisme (1832), Lucien Leuwen (1834), La Vie de Henry Brulard (1836), Les Chroniques ltaliennes (1837), Les Mémoires d'un touriste (1838), La Chartreuse, L'Abesse de Castro (1839), Lamiel (1841). Comment peut-on interpréter son aveu en tant que révélation d'une aridité qui l'accable, d'une créativité qui tarit? D'ailleurs Michel Crouzet soutient que «A-Imagination peut s'inscrire dans cet étonnant carrefour stendhalien: d'un côté cette imagination des minuties basses ou baroques, de l'autre celle des temps hérö̈ques» ${ }^{8}$.

(7) M. Molho op. cit., p. XI.

(8) M. Crouzet, Notice sur "A-Imagination" in 
On ne veut pas aborder ici le problème de l'échec de ces contes, souvent inachevés et «inachevables» (M. Crouzet). Cela appartient à un autre genre d'analyse. Des romans ratés? Des exercices, des ébauches? Oui! Mais la question qu' on se pose ici est la suivante: pourquoi l'Espagne dans cette toile de fond? Pourquoi l'Espagne picaresque? C'est l'image d'une Espagne parfois ouvertement annoncée, parfois perçue ça et là, mais indiscutablement présente, même lorsque l'action se déroule ailleurs. Toujours les mêmes facteurs déterminants: mendicité, gueuserie, corruption... Les protagonistes tour à tour rusés ou idiots et souvent, l'a-t-on déjà dit, victimes et bourreau. Du moralisme sous-entendu et qu'on comprend par le négatif. Toutefois ces personnages ratés sont inoubliables car ils sont éloquents.

Encore quelques détails sur l'image du picaro espagnol pour mieux découvrir les traits qui peuvent avoir frappé Stendhal:

1) Picaro ( dérive du verbe picar = piquer ou picorer).

2) Un picaro s' exprime à la première personne, autrement nul autre ne parlerait de lui. Ce personnage-héros ne fait que raconter rétrospectivement. Dans ce jeu rétrospectif il y a souvent amertume et le récit prend parfois un aspect fragmentaire et disparate où les épisodes se juxtaposent.

3) Son obscurité est due à son lignage honteux (mère prostituée, père voleur).

4) Puisqu'il incarne l'antithèse de l'honneur, il est moins qu'un roturier, il n'est qu'un déchet social.

5) La mendicité. Le vol... les larcins. Le parasitisme... la faim: les picaros ont toujours faim, une faim rabelaisienne. Avec le parasitisme la ruse: « ... apprends, nigaud, un garçon d'aveugle doit en savoir un point plus que le diable», s'écrie l'aveugle en s'adressant à Lazare de Tormes'.

6) Le picaro n'est jamais paysan, il ne saurait pas se consacrer au travail de la terre. Par conséquent l'argent le hante. Tous les expédients sont bons pour voler, pour duper (à propos des vols, on aura, chez Stendhal, le voleur volé, sujet énormément intéressant si l'on tient compte de la duplicité des situations et de la dualité de l'âme et des tempéraments, ce qui hantait Stendhal). Don Pardo, par exemple, en est un témoignage, c'est pourquoi on l'a défini un récit picaresque (v. Victor Del Litto).

7) À travers son expérience, le picaro veut discuter la valeur temporelle et spirituelle de l'homme et dans sa lucidité il mettra en question sa personne et son destin, mais aussi la moralité et les règles sociales de ses contemporains et surtout celles de ses supérieurs. Cette tendance à juger manque dans El Buscón de Quevedo, c'est pourquoi ce roman, très connu et très intéressant, n'appartiendrait que partiellement à la littérature picaresque ${ }^{10}$.

Epuisée en Espagne avec Quevedo (La Vie de l'Aventurier Don Pablos de Ségovie ou El Buscón) la pensée picaresque ne pouvait pas renaître au XVIII ${ }^{\mathrm{e}}$ siècle. Si l'ancienne conception du lignage, strictement liée à la conception de l'honneur, était à la base de la picaresque, il faut admettre que la philosophie des Lumières, ouvrant les portes à l'égalité sociale et humaine, pose une pierre tombale sur le concept de l'antihonneur, par conséquent l'image du picaro s'efface. Cela est sûr, même si, en Espagne, la bourgeoisie, n'a pas eu le même essor qu'ailleurs. C'est la bourgeoisie qui tue le haut lignage!

(9) Romans picaresques espagnols - La vie de Lazare de Tormes, Paris, Gallimard, 1968, p. 7.

(10) Lazare de Tormes, autre roman picaresque celui-là, mais qui n'en représenterait pas le parfait exemple, bien qu'on le considère comme le premier témoignage de ce genre. L'auteur anonyme serait- il plus artiste que les autres écrivains picaresque? Peut-être que oui. À propos de cela lisons ce qu'écrit Maurice Molho: «La vie de Lazare, se présente comme un jeu d'esprit, cocasse et cruel, qui détruit toute chose et, dans le sourire se détruit lui-même». (M.MolHo op. cit. p. 40) 
La honra, l'honneur espagnol, émanerait de la nature même de l'homme, mais de l'homme qui est noble en vertu de sa naissance. Le déshonneur picaresque suppose une religion de l'honneur. C'est une vision manichéenne de la vie, les bons d'un côté, les méchants de l'autre. L'honneur est car le déshonneur existe. L'honneur s'hérite ainsi que le déshonneur. Le noble appartient à la noblesse, le gueux à la gueuserie. Cette vision du monde sous-entend le malheur, c'est une acceptation du malheur des uns et du bonheur des autres, le bonheur des privilégiés. Cela dérive d'une conception de la vie sociale qui remonte à la féodalité. Plus tard les libertins français, Gassendi en tête, élaborèrent, heureusement, une explication du monde inspirée d'Epicure! Par la suite Saint-Just soulignera que «le bonheur est une idée nenve en Europe». La vie sociale change alors, et la littérature picaresque aussi. Maurice Molho écrit: «Dès l'instant qu'on érige en principe que le malheur, issu du péché originel, n'est pas la condition naturelle de l'homme, la pensée picaresque, désormais déracinée, jette bas les armes et expire» ${ }^{11}$.

C'est une ère nouvelle où s'établit un nouvel ordre des choses.

$$
* * *
$$

Mateo Alemán est juif. Le judaïsme est une condition sociale et religieuse, c'est une religion qui détermine des fractures d'ordre social, ainsi que le déséquilibre fatal qui en dérive. Filippo, Le Juif stendhalien, sera ballotté ça et là, ses revers de fortune, ses échecs seront à attribuer à ses origines. Le judaïsme est strictement lié au marchandage, aucun scrupule donc: «'étais juif» s'exclame Filippo «méprisé de vous autres chrétiens et même des juifs, car j'avais été longtemps excessivement pauvre».

Maintenant une première digression: comment trouver des liens entre le sujet qu'on est en train de traiter - ce Stendhal problématique, aléatoire - et une tradition littéraire, celle de la littérature picaresque? En plus il faut dire aussi que Stendhal ne connaît pas l'Espagne, s'est-il vraiment rendu à Barcelone? On n'en est pas sûr.

Il faudrait plutôt réfléchir sur ce que le Stendhal du Brulard écrit à propos de son célèbre espagnolisme, c'est une petite, fragile clé d'interprétation des récits tels que Le Juif, Don Pardo, Le Philtre, A-Imagination, Le coffre et le revenant, Le Chevalier de Saint Ismier et le Ménuel de Lucien Lemwen: il aurait hérité son espagnolisme de la part de son grand-père Gagnon et de sa tante Elisabeth: «Tout l'honneur, tous les sentiments élevés et fous de la famille nous venaient de ma tante Elisabeth» [...] et ailleurs «J'avais et j'ai encore les goûts les plus aristocrates». Et encore: «Cet espagnolisme, communiqué par ma tante Elisabeth, me fait passer, même à mon âge, pour un fou de plus en plus incapable d'aucune affaire sérieuse» (H.B. Le Divan 1949- Tomel p. 233). Ces aspirations vers les hautes sphères, vers la noblesse d'âme, vers une aristocratie toute intérieure, mêlée aux tendances républicaines, supposent-elles une religion de l'honneur telle qu'on l'a remarquée chez les Espagnols de la littérature picaresque? Oui et non, car ce raccord serait à rechercher alors dans le sens de l'bonneur qui, chez les écrivains picaresques, n'appartient qu'à la caste, alors que chez Stendhal, le sens de l'honneur appartient à l'individualité de la personne.

Dans le Brulard on lit «... le conte espagnol le plus commun s'il y a de la générosité me fait venir les larmes aux yeux». Cette générosité est-elle d'ascendance cornélienne? Beau comme le Cid, c'était le grand mot de tante Elisabeth! Et la grande passion, dès son adolescence, pour le chevalier à la Triste Figure? On devrait ouvrir là-dessus tout un discours trop important et qui nous mènerait loin de notre sujet. Il

(11) Ibid, p. CXI et p. CXIII M. MolHo à propos de El Buscón parle de «... dissolution du genre

picaresque». 
vaut mieux donc se borner à ce que Stendhal écrit à propos du Quichotte: «la découverte de ce livre [...] est peut-être la plus grande époque de ma vie» ${ }^{12}$. En voilà assez sur la noblesse de l'âme et sur la capacité d'idéaliser, d'atteindre le sublime, la «folie», et en même temps d'en être dupe. Don Quichotte ne fait que sublimer la réalité. Dans le magma de sentiments y a-t-il chez Stendhal des arrière-pensées menant, subtilement, à cette Espagne rêvée, inconnue, dont l'idéalisation alterne des aspects positifs à d'autres qui ne le sont pas? Cette Espagne d'où est-elle issue? Stendhal ne la connaît que très peu! Ses observations sur l'Ándalousie au chapitre XLVII de L 'Amour, ne sont que le reflet des lettres de Giuseppe Pecchio, patriote milanais, condamné à mort en contumace en 1821. D'après les lettres que Giuseppe Pecchio envoie à une belle Anglaise, Lady J.O. Stendhal cite: «Le but des Espagnols n'était pas la gloire mais l'indépendance. Si les Espagnols ne se fussent battus que pour l'honneur la guerre aurait été finie avec la bataille de Tudela. L'honneur est d'une nature bizarre, une fois qu'il a été taché, il perd toute sa force pour agir» ${ }^{13}$.

Ailleurs on lit: [...] les grands seigneurs espagnols que nous avons entrevus à Paris ne sont pas copies. Chez eux je ne vois nullement le besoin d'être rassurés de l'opinion des cent nigauds bien vêtus». Et puis Stendhal fait parler M. Ipol, philosophe catalan, dit-il, qui s'intéresse au langage du peuple: «Il (le langage du peuple) est près de la nature, il est énergique [...] À Barcelone un arrieros (muletier) m'enchante par son langage, sa personne me plaît; c'est un grand garçon fort, vigoureux, rempli d'une énergie sauvage (c'est nous qui soulignons) dont la vue réjouit l'âme. À coté de lui qu'est-ce qu'un grand d'Espagne? [...] Plusieurs de mes compagnons ressemblent tout à fait à Don Quichotte; c'est la même loyauté et la même absence de raison [...] Les cordes qu'il ne faut pas toucher, c'est la religion ou les privilèges de la noblesse. Ces messieurs me prouvent sans cesse, avec beaucoup d'esprit et une vivacité charmante, que les privilèges de la noblesse (c'est nous qui soulignons) sont utiles au peuple» ${ }^{14}$.

Les expressions telles que honneur, énergie sauvage, loyauté, privilèges de la noblesse sont ici soulignées, pour une comparaison - qu'on fera par la suite - avec certains traits caractérisant la littérature picaresque.

Stendhal semble donc céder à des sollicitations qui lui dérivent de la lecture des romans picaresques espagnols, parfois filtrée à travers Scarron ou Le Sage.

On pourrait se livrer à des conjectures: le Stendhal avide d'apprendre et de connaître peut tout «combiner» au niveau subliminal de sa conscience. Dans la littérature où l'imagination joue son rôle, il y a des lignes de force qui sont à la base de l'imagination créatrice. À cela on pourrait ajouter que les constantes psychologiques, ayant longue durée, s'étayent les unes les autres, celles de l'adolescence et celles de la maturité, à partir d'un générateur initial: le jeune Beyle se nourrit des idées nobles de sa tante Elisabeth et de son grand-père Gagnon, ce sont eux qui lui apprennent à aimer tout ce qui est noble, élevé, supérieur, aristocratique. De là l'espagnolisme, cet espagnolisme qui fait souffrir l'adolescent ainsi que l'homme de l'âge mûr. L'Italie est toujours présente chez Stendhal, alors que l'Espagne, en tant que réalité vécue, n'apparaît que rarement. L'Espagne n'est que métaphore! Elle n'est que réalité métonymique. Dans un transfert de sens, où le terme concret revêt une abstraction, par substitution analogique on peut définir l'Espagne stendhalienne une sorte de «patrie du cœur» où règnent la fierté et l'bonneur, mais aussi l'énergie sauvage, et les privilèges de la noblesse. Cela qui devait avoir son lien avec les couches sociales du milieu pica-

(12) Stendhal, Vie de H. Brulard, Paris, Gallimard, p. 106

(13) Stendhal, De l'Amour, Paris, Garnier,
1959, Notes, p. 459.

(14) StendHal, Voyage en France, Paris, Gallimard, 1992, p. 569. 
resque étant donné la hiérachie de l'époque. L'Espagne donc, grande métaphore, est la terre de la noblesse d'élite.

L'Espagne, patrie du picaro, n'en serait qu'un autre aspect, non pas le revers d'une même médaille, mais une présence, rien qu'une présence attirant l'écrivain plus que l'homme, celui-ci serait fasciné par l'Espagne, terre de la noblesse d'élite. L'Italie, au contraire, est un mythe, l'Italie... c'est le coup de poignard et l'énergie, mais c'est le clos des palais aussi, c'est le pays des tours où la solitude offre de grandes possibilités, on y réfléchit, on y rêve. C'est un pays qui naît de l'imagination.

La hiérarchie espagnole devait intéresser le Stendhal écrivain. Par conséquent on est tenté de voir un lien concret reliant le Stendhal des récits courts aux romans de la littérature picaresque.

Cette tentative qui comprend aussi l'influence du Roman Comique de Scarron (1651-1657) et Gil Blas (1715-1735) de Le Sage, est problématique, mais très intéressante. Là aussi les rythmes, le débit du discours, malgré ses ruptures et décalages, semblent revêtir parfaitement la narration, qui n'est jamais sèche, cette narration tourbillonnante, luxuriante et, surtout, riche en images. Scarron vit de littérature picaresque si ce n'est qu'indirectement. Il est réaliste, caricatural, brutal, mais non strictement picaresque. Dans la dédicace, pourtant, Scarron adresse une exhortation au Cardinal de Retz: «Ô lecteur bénévole». C'est une captatio benevolentiae par laquelle Scarron sournoisement et candidement à la fois, espère trouver bon accueil. S'agit-il d'une candeur feinte? Voulue? Est-ce un leurre?

Cette même exhortation se trouvait dans le Prologue de Lazare de Tormes (... je vous supplie, Monsieur, de recevoir cette pauvre offrande de la main de votre serviteur...) et aussi dans celui de La Vie de Guzman d'Alfarache de Mateo Alemán (... je ne doute point du généreux courage de Votre Seigneurie, dont il suffit qu'elle déploie les ailes de sa coutumière clémence, pour qu'à leur ombre mon livre se délivre de ses calomniateurs).

C'est une débauche de couleurs où l'habileté linguistique est débridée. Cela cessera à la fin du XVIII ${ }^{e}$ siècle.

Dans Gil Blas Le Sage, lui aussi, s'adresse au lecteur et lui dit «ami lecteur, tu y trouveras, l'utile mêlé avec l'agréable»; à ce préambule suit l'autre qui est adressé «Au vulgaire», puis un autre «Au prudent lecteur: «Lis de ton mieux ce que tu liras, ne ris point de mes devis et prends garde d'en laisser échapper les avis. Fais bon accueil à ceux que je t'offre, et à l'intention qui me les fait donner». Et enfin une «Brève déclaration pour l'intelligence de ce livre».

Il est évident que le souci des auteurs est fort, l'ouvrage est accompagné de l'auteur, ainsi que le démarrage d'une entreprise. Ces préfaces, dédicaces, placet et préambules sont fréquents chez les écrivains espagnols du XVII ${ }^{e}$ siècle qu'on définit picaresques et chez d'autres auteurs européens y compris les Italiens. Mais ce qui nous frappe c'est qu'on trouve cet usage chez Stendhal aussi. Est-ce une mièvrerie? Ce n'est pas seulement dans les récits courts, dont on a parlé, qu'on trouve ces expressions-là! On lit «Ô lecteur bénévole» dans Lamiel (p. 53), dans Lucien Leuwen (p. 767) «Lecteur bénévole, écoutez le titre que je vous donne. En vérité si vous n'étiez pas bénévole...».

$\mathrm{Au} X \mathrm{XVII}{ }^{\mathrm{e}}$ siècle, l'usage de ces préambules, était encore en vogue mais, après le siècle des Lumières, la société du monde entier a changé; comment interpréter alors ces effusions obséqueuses chez Stendhal? La courtisanerie ne lui sied pas, ou mieux elle ne lui sied que par convenance, ou lorsque une secrète sympathie l'invite à agir. Et alors est-il influencé par Mateo Alemán, par Scarron, par Le Sage? Peut-être que oui! Malgré le travail énorme de la période 1830-1840, Stendhal devait songer, de temps en temps, à la littérature picaresque ou à d'autres romans tels que par exemple, Gil Blas de Le Sage. Stendhal semble transposer quelques échos de l'atmosphère 
lugubre espagnole dans ses récits tels que Le Juif, le Philtre, ou bien Don Pardo etc. C'est une atmosphère lugubre mais mouvementée où le pouvoir de certains puissants est souvent fondé sur la faiblesse des autres. Alors intrigues, complots, coups de théâtre et vols, vols à répétition, sont la règle, ainsi que les commerces illicites.

\section{Le Juif}

Dans Le Juif, Filippo, qui s'exprime à la première personne, se laisse saisir par les événements son tempérament n'étant ni faible ni fort; il n'est jamais maître de la situation bien qu'il arrive à en tirer quelques avantages. Trompeur, il l'est, mais jamais hypocrite... cela est très singulier surtout si on réfléchit sur l'attention que Stendhal vouait à l'hypocrisie. En même temps on remarque chez Filippo une légèreté d'esprit incroyable. Filippo est toujours en quête d'argent, et... très légèrement il se débrouille, il sait toujours se tirer d'affaire. Il faut souligner que Lamiel, par exemple, est un roman où l'intrigue est faible, alors que l'héroïne possède un caractère fort. Filippo, au contraire, étant totalement absorbé par l'intrigue, en est presque "étranglé", "englouti" même, hélas! Il saura sortir, pourtant, des mailles de l'ennemi. Sa seule passion reste toujours l'argent. Il incarne l'antithèse de l'honneur à cause de ses origines, mère et sœur voleuses, lui aussi voleur et victime de sa mère, son beau-père lui aussi voleur ainsi que ses beaux-frères. Un fameux avocat de Padoue, devenu aveugle, le fait entrer à son service et le protège. D'autres aventures (où l'argent est toujours présent) nous permettront de retrouver notre héros à Lyon où il fait la contrebande, et puis à Valence où il rencontre son ami Bonnard. C'est ici qu'il entreprend l'aventure sentimentale avec Catherine, la sœur de son ami. L'esprit de commerce domine les sentiments, son ami Bonnard lui offre sa sœur Catherine «[...] n'as-tu pas laissé à ta femme de Zara toutes tes marchandises? Qu'elle vive, elle à Zara et toi reste avec nous!» La diaspora du monde antique se renouvelle dans l'Europe du XIX ${ }^{\mathrm{e}}$ siècle. Filippo n'est qu'un "pauvre juif d'Innsbruck". Tracasseries et vols, vols à répétition. On l'apostrophe «chien de juif» et une «terrible malédiction» pèse sur lui. C'est Catherine qui, belle et innocente, lance sa malédiction contre ce pauvre Filippo, lequel voudrait rentrer à Zara où l'attend sa femme Stella. Un coup de couteau clôt le récit, mais ce meurtre, dont Filippo se souille, ne révèle que l'échec total, final et fatal de ce petit juif qui n'est ni bon ni méchant, mais un peu rusé, mi-rusé mi-naï, un peu comme le père de Guzman d'Alfarache qui à l'église «... tous les matins (il) oyait la messe à deux genoux, les mains jointes, hautes et son chapeau dessus. Les médisants y trouvèrent à mordre, arguant qu'il priait de la sorte pour ne pas ouïr le service, et qu' il portait le chapeau haut pour n'en point voir la célébration $\gg^{15}$. Cela veut signifier qu' il était resté juif tout en affichant une dévotion extérieure.

L'ouvrage de Mateo Alemán est pénétré d'un fatalisme intégraliste typiquement hispanique, contre-réformiste: «La lignée du picaro, écrit M. Molho, déshonorée de génération en génération, participe en fait d'un déshonneur radical, qui est celui de l'humanité déchue» ${ }^{16}$. Toujours le péché originel, ce qui ne pouvait intéresser Stendhal qu'indirectement, mais un reflet en pourrait être la malédiction qui semble menacer Filippo. Nous disons «qui semble» car cet épisode de la malédiction, à notre avis, est nuancée d'ironie, une ironie débonnaire... exceptionnellement débonnaire chez Stendhal. 


\section{Le Coffre et le revenant (9 Mai 1830)}

Le 8 septembre 1829 Stendhal quitte Paris pour se rendre à Bordeaux, Toulouse et peut-être à Barcelone. Le 9 mai de l'année suivante il publie Le Coffre et le revenant dans la Revue de Paris. Le sous-titre est Aventure espagnole.

Le décor est totalement espagnol: les protagonistes issus d'une hiérarchie rigoureuse vivent leur histoire autour et dans l'ancien palais de l'inquisition de Grenade où habitent le vieux don Blas et sa charmante femme Inès. Celle-ci est aimée à la folie de don Fernando, blond et beau comme le soleil.

La camériste Sancha, personnage vaguement picaresque à cause de sa condition sociale et de ses traits caractéristiques, devant la fureur de don Blas, qui se croit trahi, révèle un «regard espagnol, mélange singulier de crainte, de courage et de baine». C'est encore le Stendhal qui sonde les profondeurs de l'âme à travers les plis et les replis du corps.

Tout cela est très intéressant mais il n'y a rien de picaresque dans toute cette nouvelle. Il y a ce coffre, où se cache Fernando poursuivi par don Blas, qui pourrait renvoyer aux récits picaresques, surtout dans ses ballottements macabres au milieu d'un cimetière. Mais c'est peu de chose. Tout au plus on pourrait y voir du picaresque théâtral. Le style est léger et désinvolte.

Ce qui manque c'est le personnage du picaro et son milieu. Aucun raisonnement aigu et sournois. Est-ce une Espagne que Stendhal a créée après son voyage à Barcelone?

\section{Le Philtre}

Le Philtre qui avait paru en juin 1830 dans La Revue de Paris a le sous-titre suivant Imité de l'italien de Silvia Valaperta. Ce n'est qu'un alibi, l'auteur imité n'est pas italien, mais français. C'est Scarron. L'ouvrage imité est L'Adultère innocente. «Je voulais me désennuyer d'un fort grand mal de tête, écrit Stendhal, de là le plagiat dont j'ai averti La Revue [...] Le tout sans aucune intention de publier» ${ }^{17}$.

L'action se déroule à Bordeaux, c'est une histoire d'amour qui n'a presque rien de l'atmosphère picaresque même s'il y a des coups de thêâtre, du butin volé, de bizarres superpositions de personnages, lesquelles, loin du jeu de miroirs de la picaresque où un personnage se superpose à l'autre en s'observant réciproquement, confèrent du mouvement à la nouvelle en étayant la théâtralité. Dans A-Imagination on retrouvera beaucoup plus intelligiblement ce jeu de miroirs.

Le Philtre est une nouvelle qui a joui du plus grand succès. Le protagoniste se débat entre le comique amer, l'aventure rocambolesque et son idéalisme romanesque.

Ce récit pourrait être utilisé pour la scène.

\section{Ménuel (1836)}

Histoire du lancier Ménuel.

Cet épisode ne figure ni dans le manuscrit de Lucien Lenwen ni dans la copie dictée en 1835.

Stendhal l'écrivit de sa main à Paris les 6 et 7 Septembre $1836^{18}$, sur le manuscrit

(17) Stendhal: Le Rose et le Vert. Mina de Vanghel et autres nouvelles, Paris, Gallimard, 1982, p. 484.
(18) V. L. Leuwen. Notes et variantes in Romans et Nouvelles, Paris, Gallimard, 1972, pp. 15011502 . 
primitif, mais en marge. À la fin de l'histoire de Ménuel on lit «ces huit pages sont un épisode».

Ménuel: encore un jeune vagabond, avec son histoire de pauvre diable. «[...] honni, bafoué dans toute la ville», il est condamné aux galères pour un faux. On le voit se déplacer de Pau à Bayonne, puis à Saint Sébastien... Il raconte sa vie aventureuse: «[...] Je suis un grand misérable, faussaire, condamné aux galères et lâche».

Encore le Mal. Toujours le même univers irréel et hyperbolique, toutefois la dépravation, ici, n'est pas excessive, ce sont des scènes fortes, mais on les décrit d'un ton léger. Par conséquent la débauche n'a rien de solennel, ainsi qu'il arrive, par exemple, dans $A$-Imagination où les gestes de l'abbé Richet semblent être presque hiératiques.

Cet épisode est détaché du reste du roman. Est-ce un récit secondaire? C'est ici que réside l'importance du récit. Est-ce un des procédés permettant de mieux structurer la trame narrative? Il pourrait représenter, par ailleurs, un essai sur le genre romanesque et sur la façon de raconter.

«Les récits secondaires, écrit Marc Marti, sont au service d'une littérature qui, tout en revendiquant l'exemplarité, n'en oublie pas moins de distraire le lecteur» ${ }^{19}$.

\section{A-Imagination}

Maintenant A-Imagination, récit que Stendhal écrit en 1838. Ce titre, visiblement, sémiologiquement moderne, appartient au langage visuel. Ailleurs aussi, très souvent, au gré de son caprice et de sa fantaisie, Stendhal s'est évertué à jouer avec les mots, les combinaisons multilingues étant à l'ordre du jour. Ce titre possède quelque chose de très singulier (il semble même vouloir nous renvoyer aux avant-gardes).

Dans une note marginale on lit «Je ne sais où je vais» 2 août 1838. La fragilité, la précarité de la situation sont bien évidentes.

A-imagination désigne une personne sans imagination, mais c'est dans cette totale absence d'imagination que Robert, le protagoniste, arrive à inventer des tours pour parvenir à la fortune.

Dans A-Imagination Stendhal semble introduire vraiment ce jeu de miroirs qu'on trouve dans Guzman d'Alfarache (on pourrait se rapporter à Gil Blas aussi en ce qui concerne ce jeu, mais Gil Blas n'est pas un picaro au sens strict du terme. Toutefois il faut dire que, parfois, il y a des dédoublements à cause du caractère rétrospectif du récit).

Lisons ce que Maurice Molho écrit à propos du jeu de miroirs dans Guzman d'Alfarache [...] Guzman se dédouble donc en deux êtres, dont l'un est la négation de l'autre, séparés par le seuil, déjà franchi lorsque s'ouvre le livre, d'une conversion annoncée à plusieurs reprises et amorcée dans les dernières pages [...], mais ce qui est étonnant, c'est que «dès la première page du livre, avant même que le récit ne commence, la donnée picaresque s'est inversé dans Guzman [...] Celui-ci «se transcende pour se décrire et oppose à l'immanence picaresque un au-delà à partir duquel il se juge [...] Il s' ensuit que Guzman d'Alfarache [...] se présente comme un roman picaresque à l'envers, où le picaro n'existe que dans la mesure où il devient son propre antagoniste» ${ }^{20}$.

Dans A-Imagination Stendhal suit un procédé qui, malgré l'inachèvement du récit, malgré les nombreuses fragmentations des concepts, semble avoir des analogies

(19) Marc Marti: Nouvelles intercalées et récit secondaires, dans Le roman espagnol du XVIIIe siè- cle, in «Les langués néolatines», sett. 2001, p. 18.

(20) M. MolHo. op. cit., p. XLVIII. 
avec les procédés de Mateo Alemán. C'est une sorte de stratagème où des images reflétant les traits caractériels d'un personnage, reproduiraient ces mêmes traits dans un autre personnage ... et dans un autre encore. Parfois, dans son plan préparatoire, Stendhal semble tenter une sorte de chassé-croisé, bizarre échange réciproque, où Robert Macaire, le héros-protagoniste, devrait ressembler selon la volonté du père, à l'autre Robert Macaire, ce type d'homme d'affaires escroc, célébré par Daumier et par Frédérick Lemaître. Cela, écrit Gilles Louÿs, «indique un projet romanesque de grande ampleur» ${ }^{21}$.

Par la suite c'est l'abbé Richet qui joue la grande scène: il voudrait faire de Robert un individu tantôt subjugué et dominé tantôt pervers et dominant. L'abbé Richet, homme à imagination, veut-il construire un automate à l'image inverse de lui? «[...] Robert empaume de plus en plus cet abbé dont l'admiration est ravie par l'absence de cœur et d'imagination de Robert». C'est du trompe-l'œil! C'est la duplicité de la vie où fausseté et hypocrisie jouent leur rôle. Ou bien, les «artifices de perspective» sont-ils employés pour ne donner que l'illusion de la réalité? La conscience de la multiplicité de la réalité et l'exigence de l'introspection sont toujours très fortes chez cet écrivain attiré par le masque (qu'on pense au touriste-marchand de fer des Mémoires d'un touriste) ainsi que par l'atomisation des sentiments, des passions et des instincts.

C'est compliqué! Pourtant le romancier connaît et utilise les effets de distanciation par rapport à l'action et par rapport au lecteur. Certes, il s'agit toujours d'un conte inachevé et d'un plan préparatoire, mais on peut quand même entrevoir quelques lignes directionnelles.

On a parlé d'images reflétant des traits caractériels, ces reflets miroitent des qualités et des vices ainsi que des nuances. C'est l'insaisissable des situations. Ces contes souvent, sont narrés rétrospectivement. Cela semble confirmer ce que, au XX $\mathrm{XX}^{\mathrm{e}}$ siècle, plusieurs critiques ont affirmé à propos de la technique romanesque de Stendhal: Moravia en tête parlait souvent d'un Stendhal écrivain cinématographique.

La mécanique des personnages est compliquée et simple en même temps. Est-ce du matérialisme mécaniciste? Stendhal monte et démonte des pièces. Se trouve-t-il dans la situation de celui qui ressent le besoin de se frayer un chemin dans un panorama inexploré? On ne saurait pas répondre. Dans le plan du 2 août 1838 on lit: «Robert emploie cette heure à apprendre le droit chez le procureur, le plus pauvre du Palais de justice. Il aime à voir juger un homme accusé du crime capital. Lorsque l'accusé est innocent, il se retire dégoûté. Raconter cela en action, ne pas le dire». Cette phrase que Stendhal adresse à lui-même est incontestablement révélatrice, c'est une sorte de credo de sa composition littéraire. Voilà donc, à notre avis, la clef d'interprétation de tous les récits que nous venons d'analyser, y compris celui ci, lequel ne présente pas qu'un intérêt philologique, d'origine picaresque. Ici on a la vraie révélation de Stendhal en ce qui concerne la narration. C'est une révélation qui n'est pas une nouveauté, c'est du déjà vu, mais on est tenté de supposer que, tous ces récits, y compris les plans, ne révèlent que l'importance du réalisme de l'action - FAIRE PARLER L'ACTION - Stendhal brise le moule du récit court «... il ne donne que le squelette de certains passages, écrit Henri Martineau, qui sembleraient, à priori, d'une importance capitale et il s'étend à loisir sur d'autres scènes qu' il gonfle hors de toute proportion».

Le romancier donc y crée l'indicible, l'impalpable, l'invisible, il crée une situation de laquelle dérive l'abstrait... C'est l'abstrait qui donne un sens à l'action: dans 
une note du plan de A-IMAGINATION, vers la moitié de son travail préparatoire, Stendhal, à propos de la formation du caractère du protagoniste, annote: «Si j'évite l'odieux, j'accroche le comique: c'est un Gil Blas nouveau». Stendhal lisait Lesage à l'époque, mais le personnage stendhalien est beaucoup plus abstrait que celui de Le Sage, qui doit beaucoup à ses modèles espagnols. Dans Gil Blas l'auteur narre les multiples aventures d'un vaurien très sympathique, qui est devenu fripon à cause de l'injustice sociale. C'est la société française, une société hypocrite qu'on pourrait retrouver chez le Stendhal qui fera dire à Robert: «Il faut être tout autre chose que dévot pour écarter le soupçon de friponnerie, ces gens-ci se croient dévots mais la religion ne les amuse pas assez, ce qui les amuse c'est la Bourse».

Le Robert stendhalien et Gil Blas ont des points communs, tous les deux sont comiques et un peu grotesques, mais Robert n'aura qu'une physionomie d'«apprenti perruquier». Au début du récit Stendhal écrit: «Il (l'auteur) prétend faire Robert absolument sans imagination autre que celle qui sert à inventer des tours pour parvenir à la fortune».

Le picaro chez Robert: du point de vue physique il fait songer aux picaros espagnols: «Robert était assez grand, fort, maigre, la figure longue, peu de menton, les cheveux d'un blond sale, les yeux gris-vert, au total la physionomie d'un chat. L'air faux et presque menteur [...] physionomie d'apprenti perruquier».

Robert doit inventer des tours car il est sans imagination: il a 14 ans... petit coquin complet il «vole des bonbons aux étalages des petits marchands avec Carière, son camarade âgé de 16 ans[...] Carière est bâtard d'une femme de chambre voleuse». (p.332)

Dans le plan du 2 août 1838 on lit «l'abbé Richet était grand et gros[...] le front fuyant, de très beaux yeux sans aucune expression outre que celle de l'égoïsme[...] un observateur fin y eût trouvé de la cruauté et aussi l'égoïsme surnageant [...] Une demiheure après le déjeuner, l'abbé s'enferme, tire au sort l'énoncé d'une question à prouver, monte dans une chaire élevée de six pieds qu'il s'est fait faire et prêche devant six rangs de chaises occupées par des mannequins vêtus [...] et au ventre de paille. Il dit un pater en chaire et improvise aussitôt un sermon. Il habille Robert en moine et se fait appeler mon oncle. Il est exactement le contraire de Robert, l'un est la négation de l'autre. L'Abbé se fait escorter par Robert et lui commande de perdre ses façons de portier, ce qui froisse Robert énormément. Les deux ne s'entendent plus, ou bien moins que jadis, Robert dit des mensonges - l'abbé a horreur de cela, Robert qui le crois un hypocrite complet ne comprend point ces sentiments». Les actions se superposent sans cesse, on n'a donc pas un tableau exact, précis, mais on a l'impression que dans Robert, dépourvu d'imagination, se reflète l'Abbé Richet, homme doué d'imagination mais égoïste et pervers, comme un homme à imagination. Il est hypocrite, il cherche donc en Robert des qualités telles que l'absence de cœur et l'absence d'imagination.

Ce qui est intéressant c'est cette sorte de jeu de miroirs qu'on a déjà cité et qu'il faut approfondir et compléter: «Guzman se dédouble en deux êtres, écrit Maurice Molho, dont l'un est la négation de l'autre, séparés par le seuil, déjà franchi lorsque s'ouvre le livre, d'une conversion annoncée à plusieurs reprises et amorcée dans les dernières pages. [...] Du protagoniste au narrateur il y a solution de continuité...» mais ce qui est étonnant c'est que «...dès la première page du livre, avant même que le récit ne commence, la donnée picaresque s'est inversée dans Guzman...». Celui-ci «se transcende pour se décrire et oppose à l'immanence picaresque un au-delà à partir duquel il se juge [...] Il s'ensuit que Guzman d'Alfarache [...] se présente comme un roman picaresque à l'envers, où le picaro n'existe que dans la mesure où il devient son propre antagoniste» ${ }^{22}$. C'est cérébral, on y trouve aussi l'esprit des agudezas, c'est la pensée qui est exprimée selon des modèles typiquement espagnols où le gongorisme 
semble laisser encore ses traces. Chez Guzman le picaro disparaît, il deviendra un conversos, car c'est la religion qui le sauve. Chez Robert le picaro s'efface au fur et à mesure qu'il se modifie à côté de l'abbé Richet... et ici aussi la religion joue son rôle, mais c'est la religion des abus des supérieurs commis à l'égard des enfants destinés à être honteusement exploités. Robert est-il nigaud? On ne le dirait pas! Par la suite on saura qu'une petite actrice du boulevard aime Robert à la folie car elle trouve qu'il n'est pas niais, le jeune homme sera «électrisé pour la première fois de sa vie par l'imagination de cette jeune fille». Robert deviendra-t-il habile et rusé? Peut-être que oui, mais l'inachèvement ne permet pas de le savoir.

\section{Le chevalier de Saint-Ismier (22 Avri11839)}

En 1951 Pierre Martino pensait découvrir la source de cette nouvelle dans un épisode de Gil Blas, «Histoire de Don Alphonse et de la belle Séraphie». Mais en 1962, grace à des études plus approfondies, on découvrit que ce n'était pas Le Sage qui avait inspiré Stendhal, mais Tirso de Molina. Il s'agit d'une adaptation libre, en langue française, du premier tome du Toledan (1654), lequel n'est que l'adaptation de Los Cigarrales de Toledo du même auteur. Los Cigarrales sont les jardins des environs de Tolède; un peu comme dans le Décaméron, on y raconte des aventures, il y a aussi des considérations importantes sur le théatre de Lope de Vega et sur sa technique dramatique.

Ce fut l'hispaniste français André Nougué qui, en 1962, découvrit cette source du récit stendhalien. Stendhal possédait ce livre, ses gloses sont innombrables mais très difficilement déchiffrables.

Lisons l'incipit de cette nouvelle: «C'était en 1640: Richelieu régnait sur la France plus terrible que jamais [...] on trouvait chez le paysan, chez le noble, chez le bourgeois une énergie que l'on ne connut plus en France après les soixante-douze ans du règne de Louis XIV. En 1640 le caractère français osait encore désirer des choses énergiques» (c'est nous qui soulignons). C'est le Stendhal qu'on connaît: l'individualité qui s'affirme par l'énergie (passions puissantes, vie dangereuse). À Bordeaux ce Chevalier de Saint-Ismier, devra se battre en duel, en voici le récit: «[...] le comte para le coup en relevant l'épée du chevalier qui lui entra dans l'œil droit, pénétra de six pouces; et le chevalier sentit son fer arrêté par quelque chose de dur; c'était l'os de l'intérieur du crâne. Le comte tomba mort». Un roman de cape et d'épée, hélas! L'énergie tourne au ridicule et le réalisme semble évoquer les Cenci, mais de façon quelque peu banale et sans aucun trait tragique.

Dans sa toile de fond l'Espagne est ici «sous-jacente», et en version rocambolesque. On a la sensation de saisir quelques échos des contes aventureux qu'on lit dans Los Cigarrales de Toledo. Mais hélas! C'est une Espagne où les picaros ne sont pas présents.

\section{Don Pardo (31 Mars-2 Avril 1840)}

Don Pardo est effectivement le récit stendhalien le plus picaresque.

L'action se déroule à Civitavecchia, ville assez triste, où «les lois ne protègent que les puissants, l'homme du peuple qui ne se protège pas lui-même est méprisé et insulté par ses égaux ${ }^{23}$. C'est très amer! Dans Le Juif, dans la littérature picaresque en général et dans Guzman d'Alfarache, on a des réflexions pareilles. Là, outre la 
blessure d'amour propre de l'homme rongé par la tare de son ascendance, il y a l'homo homini lupus. Au livre II, chapitre V, on lit: «Il n'y a point d'homme pour l'homme: nous vivons aux aguets les uns des autres comme fait le chat de la souris, et du serpent l'araignée, qui, lorsqu'elle le trouve en nonchaloir, se coule le long d'un fil, s'agrippe à sa nuque qu'elle étreint étroitement, et ne le quitte qu'elle ne l'ait mis à mort de son venin» ${ }^{24}$.

Revenons à Don Pardo. C'est l'histoire du fils d'un tourneur en poulies nommé Tommaso qui n'avait pas moins de quatorze enfants; son troisième enfant, nommé Pardo, est d'une maigreur extrême que sa mère entretient en allant chercher, dans les fossés voisins de la ville, des sangsues qu'elle lui applique tous les mois; elle pâlit sa figure d'un peu de farine qu'une boulangère sa voisine lui donne par charité. C'est ainsi que l'enfant peut demander l'aumône. Pardo dévore même des chats tout crus. Il se révèle peu intelligent, mais il réussit quand même dans la contrebande. «Il a une agilité étonnante pour marcher à quatre pattes... il arrive jusqu'à la boutique d'un boucher où il mord des bouchées entières de viande crue... ses dents sont si aiguës qu'elles emportent le morceau net».

C'est le thème de la faim, cette faim typiquement picaresque, mais qui a hanté aussi beaucoup d'écrivains à partir du Moyen Age jusqu'au Victor Hugo des Misérables ou le Zola de Germinal, ils ont créé de grandes fresques humaines aux lueurs sinistres.

Ici, en outre, il y a le thème du voleur volé, thème celui-là qui est à la base du picarisme:

a) Pardo arrive à s'emparer d'un napoléon d'or qu'un voyageur a laissé tomber, il le cache dans sa bouche, il en parle à sa mère, le lendemain il ne l'a plus, c'est sa mère qui le lui a volé. À mère voleuse, fils voleur.

b) Pardo et ses amis sont à Corneto (aujourd'hui Tarquinia), petite ville près de Civitavecchia où vivent beaucoup de gens aisés. Pardo vole des pièces d'argent, les cache dans sa bouche... mais il sera obligé de les cracher parce que ses amis, véritables filous, l'y obligent. Le parasitisme engendre l'escroquerie et vice-versa.

c) Bassesses inouïes, dépravations et iniquités... mais aussi le Stabat de Pergolèse. Des prêtres s'entourent d'adolescents tantôt naïfs tantôt espiègles, mais toujours à la merci de tartuffes sataniques. Comme la plupart des autres récits celui-ci aussi est resté inachevé.

Le plan préparatoire que Stendhal a daté du 2 Avril 1840 offre immédiatement des sensations très fortes. L' écriture segmentée et frénétique nous renvoie à celle de la littérature picaresque et à Scarron aussi, plus qu'à Lesage. Dialogues, monologues et récits, souvent délirants, créent une sorte d'ivresse de l'écriture où le personnage de Casanova semble planer dessus (Stendhal en lisait les Mémoires avec plaisir). Tous les bas instincts se déchaînant les machinations infernales se multiplient.

Dépravations et iniquités à foison... et dans ce maëlstrom le Stabat de Pergolèse! Au fond du gouffre ce Stabat semble détonner... mais ces notes, aux accents élégiaques, doivent probablement leur collocation à l'acuité de celui qui aimait tant les profondeurs secrètes de la musique de Pergolèse: les atrocités alors ressortent par contraste, la raillerie est au diapason.

Ce récit se détache net de tout ce que Stendhal a écrit.

La cruauté mentale, brutale et «raffinée», la crudité du langage ainsi que les images fortes, confèrent au récit une atmosphère qui, au lieu d'être sinistre, patibulaire, est, au contraire, piquante, légère et violente en même temps. C'est du Stendhal. Mais c'est du picarisme aussi! 
Les milieux des gens d'Eglise sont-ils stigmatisés, flétris à jamais? Non! Stendhal malgré cette dérision évidente, n'a aucune intention de s'engager dans une bataille contre le clergé. Tout au plus ce n'est qu'une de ses flèches anticléricales qu'il tire en fuyant. Au fil des ans il en avait connu des coquins fieffés parmi les prêtres! L'abbé Raillane l'avait intimément touché, marqué dans l'esprit...

$$
* * *
$$

Le dernier Stendhal - qui à l'époque s'avoue «frappé de stérilité» - semble révéler d'autres soucis et d'autres attraits: est-il en train de réfléchir sur sa propre technique de la narration où vigueur et énergie sont accélérées au plus haut degré? Dans ces récits l'analyse psychologique manque, sauf quelques cas où elle est à peine pressentie. Le Stendhal héritier d'un humanisme, où sensibilité et esprit laïque se confondent, semble ne plus penser à la "connaissance du cœur humain" que son grand-père Gagnon lui avait voulu apprendre.

S'agit-il d'un changement - ou tentative de changement - sollicité par des procédés totalement inusités en France? On pense encore une fois aux nouvelles intercalées et récits secondaires, d'origine espagnole ${ }^{25}$. Stendhal pouvait en avoir eu des exemples en lisant Guzman d'Alfarache où il y a des nouvelles intercalées tantôt dérivées d'un travail d'interpolation, tantôt authentiques: la nouvelle La veuve vengeresse est narrée par Guzman lui-même, elle est donc authentiquement vraie. Si Stendhal a lu ce roman, et si Gérard Genette a raison lorsqu'il soutient que «le véritable auteur du récit n'est pas seulement celui qui le raconte, mais aussi, et parfois bien davantage, celui qui l'écoute ${ }^{26} \gg$ (et celui qui le lit, puisque ces récits sont écrits pour être reproduits ailleurs), l'auteur de Lucien Lewwen pourrait avoir capté cette idée et pourrait avoir inséré dans son roman l'épisode de Ménuel. Cet épisode, qui a été défini conte picaresque, n’a aucun lien avec le contexte. Par conséquent il est là par la volonté de l'auteur. La passion de celui qui raconte, l'allure du rythme mouvementé, les nombreuses mésaventures du héros, font «entrer dans la régularité française - comme le dit $\mathrm{M}^{\mathrm{me}}$ de Stael - un peu de sève étrangère». Stendhal à l'époque se «ravitaillait» de tout genre de lectures, depuis les Espagnols jusqu'à Fielding dont il relut Tom Jones en 1838.

Ces récits devaient-ils offrir du plaisir au lecteur? Devaient-ils distraire le lecteur? La lecture du roman devait être agréable. La lecture, quelle fascination! Elle a toujours séduit Stendhal. Curieux de tout, les livres sollicitaient sa soif de connaissance et... peut-être son désir de trouver du nouveau. À Civitavecchia il s'ennuyait, cette ville morne ne pouvait lui offrir que des fouilles, ou la chasse aux alouettes dans la campagne romaine... mais dans sa bibliothèque il avait la possibilité de satisfaire sa soif d'évasion. «Le romancier - écrit Jaques Laurent - a besoin d'électricité qui circule entre ses repères et un inconnu qu'il a besoin de construire» ${ }^{27}$. Il faut ajouter que l'inachevé offre, plus que tout autre, l'appât de l'évolution, car il est œuvre ouverte.

Monsieur Del Litto a écrit qu'on connât mieux Stendhal si on lit ces récits courts inachevés. Il a bien raison. On pénètre mieux dans le tempérament de l' écrivain... et la stendhalogie s'enrichit davantage!

RITA ZAFFARAMI BERLENGHINI

siècle d'or, Montpellier 1987. RAIMUNDA ARANJO LUCENA, La novela corta dans l'Espagne de la fin du XVIe et le début du XXVIIe siècles, Paris 1987; JEAN LOUIS Brau, Fonction des nowvelles intercalées dans le roman espagnol au siècle d'or, Nice, CNA, 1991
("Cahiers de narratologie" n. 4), p. 437.

(26) G. GenetTe, Figure III, Seuil 1972, p. 267.

(27) Jacques Laurent, Magazine Littéraire, n. 191, janvier 1983 . 